\title{
OPTIMIZATION OF MANUFACTURING CONDITIONS FOR SPLINE OF DRUM CLUTCH HUB TAGUCHI METHOD
}

\author{
$\underline{\text { Joon-Hong Park }}{ }^{1}$, Seung-Gyu Kim², Seung-Kul Baek ${ }^{2}$, and Young-Chul Park ${ }^{3 *}$ \\ ${ }^{1}$ CANSMC, Dong-A University, Busan, Korea \\ ${ }^{2}$ School of Mechanical Engineering, Dong-A University, Busan, Korea \\ ${ }^{3}$ Department of Mechanical Engineering, Dong-A University, Busan, Korea \\ *Corresponding author (parkyc67@donga.ac.kr)
}

\begin{abstract}
A large variety of metal forming processes is required in manufacturing for automotive applications. Traditionally the design process for metal forming tools is based on trial-and-error and on the skill of experienced diemakers. This approach results in high development cost and long lead-times. Especially spline of drum clutch hub requires tight dimensional accuracy in its inside diameter and gear shape because it is used as the main component for the automatic transmission. In this paper, the most desirable or feasible manufacturing conditions for spline of drum clutch hub were found or determined by using Taguchi method and FE-simulation(FORGE-3D). Taguchi's optimization approach was used to obtain the optimal parameters. The significant parameters were identified and their effects on Manufacturing of spline were studied. As a results, a confirmation experiment with the optimal levels of manufacturing parameters was carried out in order to demonstrate the effectiveness of the Taguch method.
\end{abstract}

Keywords: FE-Simulation, Drum Clutch Hub, Spline, Manufacturing Conditions, Design of Experiment, Taguchi Method

\section{INTRODUCTION}

At present, most of the automobile components responsible for providing rotatory motion, such as gears and clutches, are produced through such cutting processes as hobbing, shaping, and shaving under the demand that they be highly precise, highly robust, and highly durable. However, research on component forming depending on plasticity processing methods such as drawing, extruding, and forging is being actively conducted in order to do away with the simplicity of processing methods that rely primarily on cutting processes, for the purpose of increasing the productivity of these components.

Plastic working has the advantages of bringing about high productivity, low rates of material consumption, low costs of production per product, and a high quality of the products themselves. A number of developments of higher value-added plasticity processing techniques have been made recently in order to meet the demands for higher competitive power of the products. Among those techniques, the deep-drawing processing method used in the forming of cylindrical containers that make use of thin plates whose surface area is infinitely larger than their thickness is difficult to implement particularly with regard to measurement precision and forming load. Technical developments are being made through various forms of research that utilize FEM(Finite Element Method) and a number of experiments based on basic theories on this topic.

The automobile drum clutch hub, for which this research focuses on developing the preform, is a main component that directly affects torque transmission needed for automobile movement and that is directly related to the overall quality of automobile performance. Products have been manufactured based on the recommendation for a new preliminary forming process required for developing precise products demanded by the automotive industry with respect to the preforms of drum clutch hubs that are manufactured in progressive dies.

These products are expected to increase the degree of precision in the manufacture of drum clutch hubs by 
solving the corner filling problem that has been regarded the most difficult to solve in existing deep-drawing processes. Moreover, they are expected to be applied to other deep-drawing processes to manufacture highly precise deep-drawing products.

\section{DRUM CLUTCH HUBS FOR TRANSMISSION}

The drum clutch hub, one of the key components in automobile transmission, is directly linked to the efficiency of power transmission and to the level of vibration noises. Figure 1 below illustrates a planet carrier with drum clutch hub products, the red dotted lines outlining the final drum clutch hub products. The manufacture of drum clutch hub products consists of importing all needed raw materials from Japan, using these materials to design preform through progressive forming processes, and finally producing the teeth of hub through Grob, Inc. cutting processes. The Grob, Inc. forming process is a cold rotational forming process that is used in the forming of a variety of products, including spline shafts, section steel, various kinds of gear products, pulley products, and the tubes of heat exchangers. Figure 2 below illustrates an example of the Grob ${ }^{\mathrm{TM}}$ forming process with a roll. Research results on the analysis and the optimal design of the $\mathrm{Grob}^{\mathrm{TM}}$ forming process for the manufacture of drum clutch hub products will be provided in a later research paper.

Kim et al. attempted a composite forming process through control metal flow and examined the minimization of material loss as a result of product manufacture and number of process decrease and the feasibility of a cuttingfree process with the use of Rigid Plastic FEM to present the optimal forming process depending on the effects of process variables. Hussain et al. analyzed the forming process of drum clutch hubs using CAMPform ${ }^{\mathrm{TM}}$ and proposed the conditions needed for manufacturing highquality products.

In recent research, Kim et al. proposed a new press forming process that can replace the $\mathrm{Grob}^{\mathrm{TM}}$ forming process. It may be possible for the new process to replace the $\mathrm{Grob}^{\mathrm{TM}}$ forming process in the areas of the approach angles from punch, the analysis based on operation methods in $\mathrm{Grob}^{\mathrm{TM}}$ forming, and a variety of ironing processes for $\mathrm{Grob}^{\mathrm{TM}}$ forming. However, the application of the progressive process to the manufacture of clutch hub products results in firstly, the increase in press capacity due to excessive forming load secondly, a significant rise in the number of processes with respect to certain products, depending on the shapes of those products. In particular, deep-drawing and ironing processes used in the forming of preforms are by themselves unable to bring about sufficient thickness in corner round. Thus, this research paper will propose a new shape for preforms to address this problem.

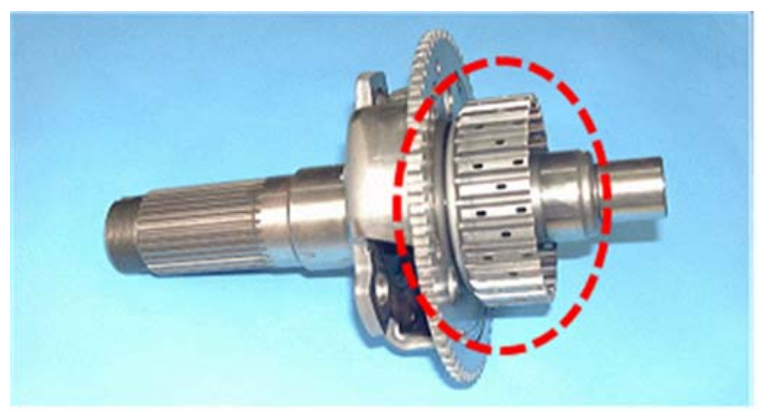

Fig. 1 Photograph of drum clutch hub products for automobile transmission

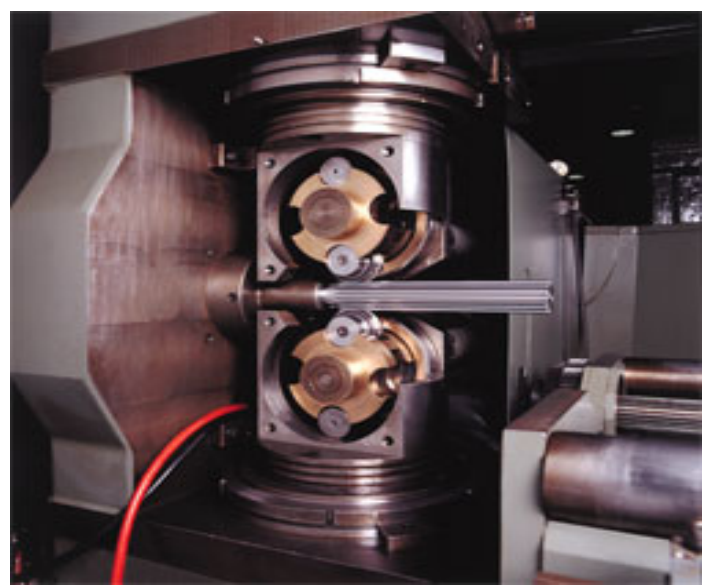

Fig. 2 An example of $\mathrm{Grob}^{\mathrm{TM}}$ forming process for spur gears

\section{DEEP-DRAWING PROCESS}

Drum clutch hub products are to be highly precise, highly robust, and highly durable. Changes in the thickness and the distribution of the wall surfaces and the floor surfaces 
of preforms whose shapes are drum-like are particularly noteworthy as they directly affect the defect in the second process, namely teeth of hub manufacture. Therefore, they are directly responsible for the qualities of the products. Much research has been conducted on the thickness distribution of products, since it is the most considerable matter of concern also in the thin-plate deep-drawing process. Among a number of research papers, those by Romanowki and Sellin have shown that the effects the shapes of corner of the die have on the distribution of and changes in thickness are the greatest.

\section{FE-SIMULATION}

DEFORM-2D ${ }^{\mathrm{TM}}$, which is a FEM code, was used in conducting a FE simulation in this research, and conditions for analysis are provided in Table 1 . One of the materials used in the manufacture of clutch drum hub preform is SAPH 3.0t, which is a key material in the production of automobile clutch components. Table 2 lists the mechanical properties of SAPH 3.0t. The following equation has been obtained from the experiment data provided in Table 2, using the flow stress curve and curve fitting.

Table 1: Condition for FE-simulation

\begin{tabular}{|c|c|}
\hline Conditions & Type \\
\hline Material of workpiece & SAPH \\
\hline Punch speed & $1.0 \mathrm{~mm} / \mathrm{s}$ \\
\hline Friction & 0.1 \\
\hline
\end{tabular}

Table 2: Tensile test result of SAPH

\begin{tabular}{|c|c|}
\hline Properties & Experiment data \\
\hline Yield Strength & $290 \mathrm{MPa}$ \\
\hline Tensile Strength & $493 \mathrm{MPa}$ \\
\hline Young's Modulus & $40.011 \mathrm{GPa}$ \\
\hline Elongation & $39 \%$ \\
\hline
\end{tabular}

\section{FORMING PROCESS}

The forming of clutch drum hub preform is carried out in two steps taking various conditions into consideration, as shown in Figure 3 This research paper proposes the reverse-drawing method in which the location and the role of the punch and the die are reversed in the general deepdrawing process. The shape of the corner of the punch and the die was selected as the process variable, as it affects the degree of measurement precision, the forming load, and the resistance to transformation the most in the first stage of the deep-drawing process. As shown in Figure 4, the shapes of the corner shape of dies and punch consist of the shape with a taper (a), the shape with a round (b), and the punch shape resulting from three types of dies with a separate taper. Depending on the shape, two steps of forming analysis in the deep-drawing process were carried out

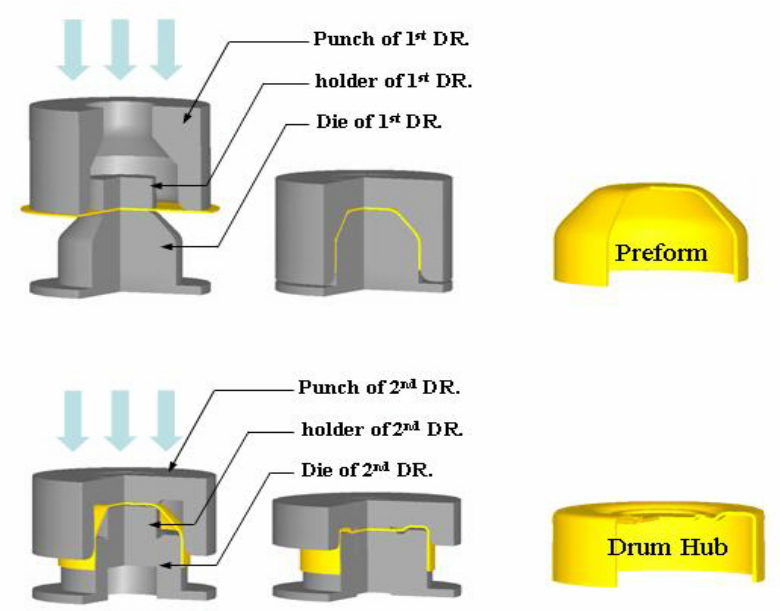

Fig. 3 Process of Deep-drawing the drum hub preform

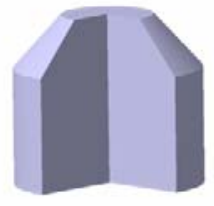

(a) Taper shape

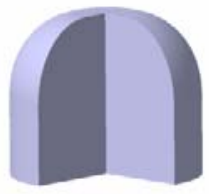

(b) Round shape

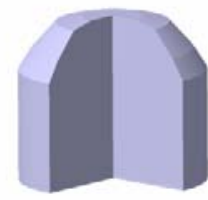

(c) Multl taper shape
Fig. 4 Various shapes of corner of die for $1^{\text {st }}$ deep drawing process

The decrease in thickness of drawing product is generally accepted to be a natural result of the execution of the drawing process. However, in the case of drum clutch hub products, designing the shape of the preform for the purpose of increasing the thickness of corner is crucial, 
since a sufficient thickness of corner is necessary to successfully carry out forming teeth of hub of the final products. This research analyzed a number of possibilities in order to ensure sufficient this thickness in deep-drawing processes, and proposes a new profile to be used in deepdrawing forming processes.

Figure 5 illustrates the decrease in the corner area in the case when the mold shape is circular in existing deepdrawing forming processes. Fig. 5 shows that the thickness of the material is decreased by at least $15 \%$ from the initial thickness of 3.0t. In order to prevent such a decrease in the thickness of the corner area, this research made use of preform with multiple tapers in carrying out the analysis. Figure 6 below shows the shape of the proposed perform of $1^{\text {st }}$ drawing.

Figure 7 shows only a $1 \%$ decrease, from a thickness of $3.0 \mathrm{~mm}$ to $2.97 \mathrm{~mm}$ of the material, in respect to the decrease in the size of the corner area. Thus, the proposed shape of the preform can be expected to prevent the decrease in the thickness of final drawing product. For the purpose of comparison, analysis as shown in the summary chart below has been performed, and its results are as follows. Figure 8 illustrates transformation phases in twostage deep-drawing processes, depending on the shape of each corner of dies and punch.

\section{THE OPTIMIAZTION OF PREFORM}

The Taguchi method was used to produce optimally designed preform with multiple tapers. The Taguchi method consists of getting data for design variables using the table of orthogonal arrays and computing the SN ratio using these data to determine the optimal value. $\mathrm{S} / \mathrm{N}$ ratio that takes into account both the mean and the distribution of the objective function, the $\mathrm{SN}$ ratio is the ratio of the expected value of the square of the population mean to the expected value of the variance. All of the steps in the Taguchi method are intended to decrease the value of the distribution. The lower the value of the distribution, the higher the $\mathrm{SN}$ ratio. The $\mathrm{SN}$ ratio is defined by the characteristics of nominal the best, smaller the best, and larger the better, depending on problems. In the forming process used in this research, the SN ratio used has the characteristic of smaller the best, meaning the lower the effective stress and effective strain that depend on material transformation, the better. In this case, the $\mathrm{SN}$ ratio is represented by equation

$$
S N=-10\left(\frac{1}{n} \sum_{i=0}^{n} y_{i}^{2}\right)
$$
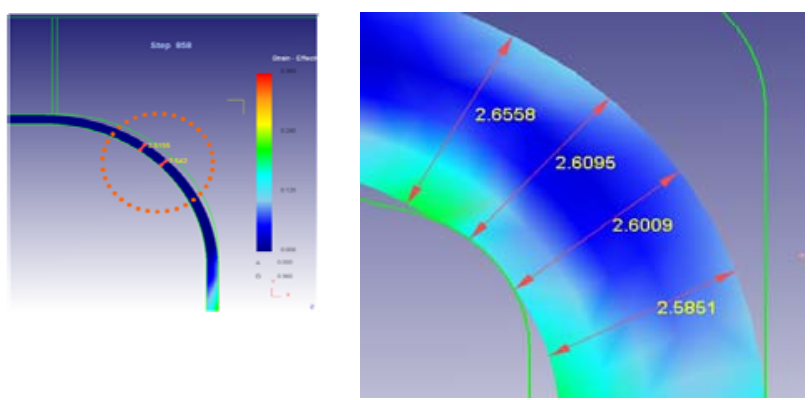

Fig. 5 Decrease of thickness dimension during the conventional deep drawing processes

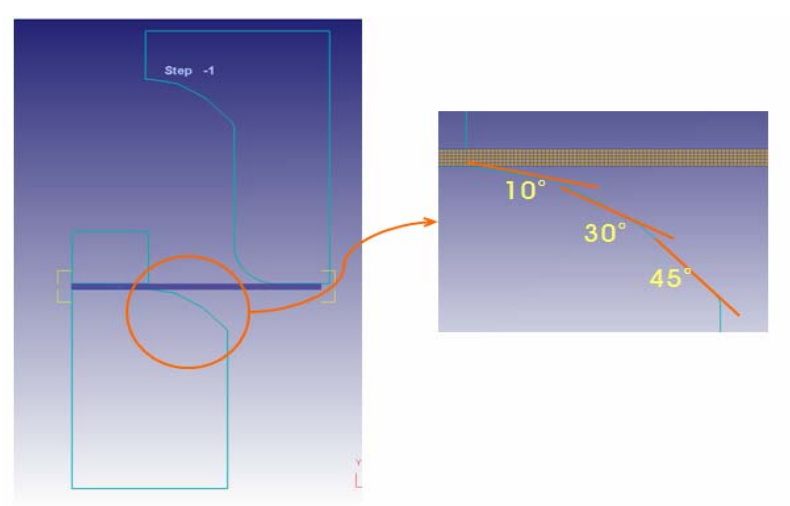

Fig. 6 The proposed profile of $1^{\text {st }}$ drawing preform

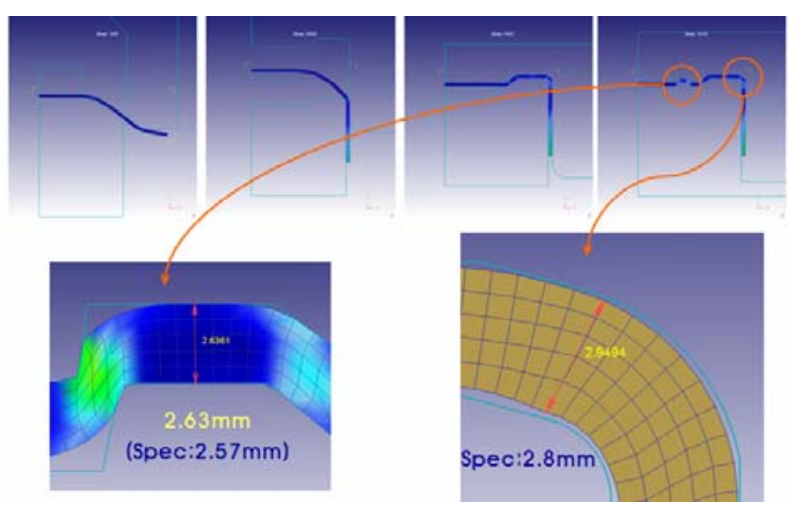

Fig. 7 Results of corner dimension after deep drawing

In equation, $n$ denotes the number of repetitions and $y_{i}$ denotes the reaction value. General sensitivity analysis 
makes use of the analysis of variance on SN ratios to evaluate the considerable effects design variables have on $\mathrm{SN}$ ratios. The reproducibility of the optimal level is evaluated in comparison with what is expected to be the optimal value, obtained from a confirming experiment. Experiment conditions are then standardized.

This research replaced the preliminary experiment with the FE simulation and took into account the 5 process variables, namely R1, R2, $\Theta 1, \Theta 2$, and $\Theta 3$ in table of orthogonal arrays L16. Table 3 lists the 5 process variables and shows the 4 levels used in this research. Table 4 provides the experiment results of L16

Table 3: Process parameters and their levels

\begin{tabular}{|c|c|c|c|c|c|}
\hline $\begin{array}{c}\text { Process } \\
\text { Parameter }\end{array}$ & Description & Level 1 & Level 2 & Level 3 & Level 4 \\
\hline X1 & R1 & 35 & 40 & 45 & 50 \\
\hline X2 & R2 & 30 & 40 & 45 & 50 \\
\hline X3 & $\ominus 1$ & 10 & 13 & 16 & 19 \\
\hline X4 & $\Theta 2$ & 18 & 22 & 26 & 30 \\
\hline X5 & $\Theta 3$ & 70 & 60 & 50 & 40 \\
\hline
\end{tabular}

Table 4: Result of L16 array with observational data

\begin{tabular}{|c|c|c|}
\hline No. & Max. Stress & Max. Strain \\
\hline \hline 1 & 469.2 & 0.7034 \\
\hline 2 & 468.7 & 0.7002 \\
\hline 3 & 470.2 & 0.6998 \\
\hline 4 & 465.3 & 0.6847 \\
\hline 5 & 468.5 & 0.6985 \\
\hline 6 & 465.8 & 0.6842 \\
\hline 7 & 461.2 & 0.7021 \\
\hline 8 & 472.3 & 0.7023 \\
\hline 9 & 471.2 & 0.7033 \\
\hline 10 & 472.6 & 0.7029 \\
\hline 11 & 466.5 & 0.7054 \\
\hline 12 & 468.1 & 0.7014 \\
\hline 13 & 467.3 & 0.6984 \\
\hline 14 & 462.3 & 0.6984 \\
\hline 15 & 469.3 & 0.6996 \\
\hline 16 & 470.7 & 0.7015 \\
\hline
\end{tabular}

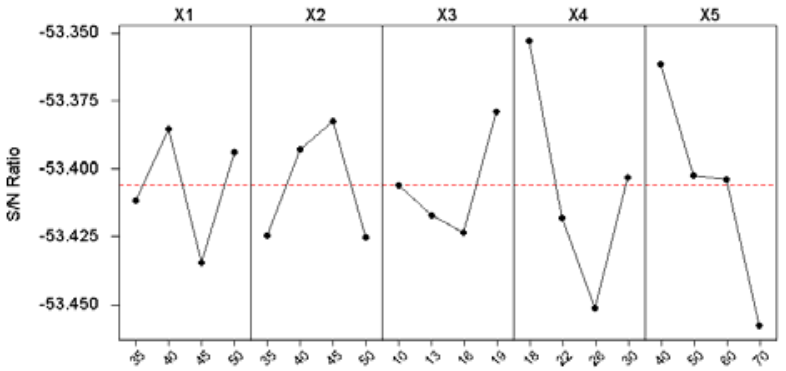

Fig. 8 Main effect plot for maximum stress

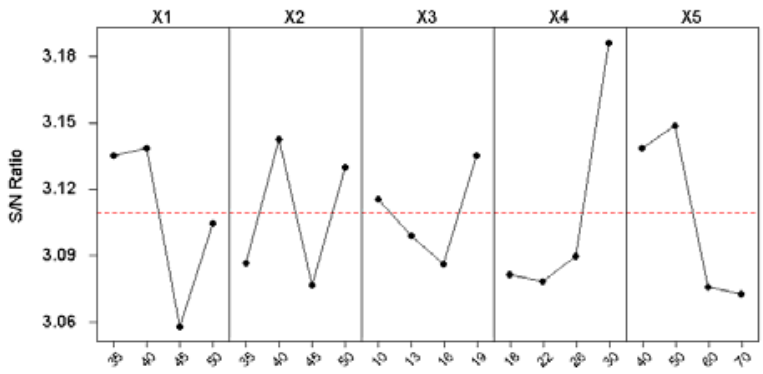

Fig. 9 Main effect plot for maximum stress

Figure 8 and Figure 9 show the main effects of SN ratios on the 5 key process variables. Evaluation of the main effects is not intended to determine an exact causal relationship by comparing with the results from the variance analysis, but to separate the incoming effect from the other effects of the reaction value in order to use the ratio of the former and latter effects in design evaluation. Overall, the characteristics of each reaction value get better as the size and level of the sensitivity of process variables decrease to Round Radius R1(X1) and Multi taper angle 3(X5) and increase to Round Radius R2(X2) and Multi taper angle 1(X3), Multi taper angle 2(X4). Thus, it can be seen that the maximum stress and the maximum strain of final product decrease when Round Radius R1(X1), Multi taper angle 3(X5) is decreased and Round Radius R2(X2) and Multi taper angle $1(\mathrm{X} 3)$, Multi taper angle 2(X4) is increased.

The process variables that have the most significant effects on hub forming and Multi taper angle 2(X4). The mean SN ratio is 2.9957 and the reaction value is 0.628 , and the 
optimal conditions of the process variables are $\mathrm{R} 1=35$, R2=50, $\theta 1=19, \theta 2=30, \theta 3=70$.

\section{DRAWING TEST}

In the shape optimization process applying the Taguchi method, it is best to select the shape shown in Figure 11 as the shape of the preform in the forming of hub products.

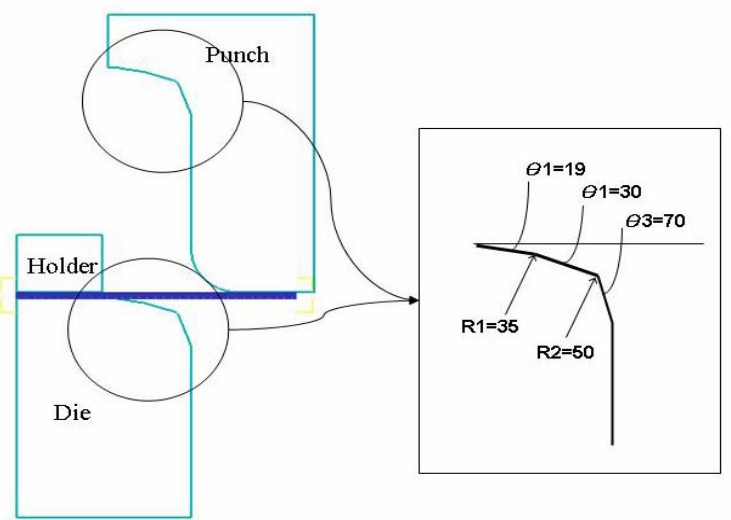

Fig. 10 Shape of optimal $1^{\text {st }}$ drawing punch and die Based on this process design, a mold has been made and preforms identical to that in figure. Figure 11 illustrates cross sections of the product, and compares the sizes of "A" “ $H$ " with actual results. Table 5 shows the results of the analysis of the thickness of each part in Fig. 12 and the results of the measurement of products. The result was satisfactory, with an error range of $+-3.5 \%$.

\section{CONCLUSION}

This research proposed a new preform shape that enables the prevention of the decrease in corner-area measurements during the 2-stage deep-drawing process of automobile clutch drum hub performs. In addition, this research focused on the effects the resulting molds, namely the shape of coner of the lower die and the upper punch, have on the thickness of the corner area of the product. The results of the process design were then taken into consideration to produce molds and carry out experiments for the manufacture of products and succeeded in making products of high quality. The conclusions from this research are as follows.

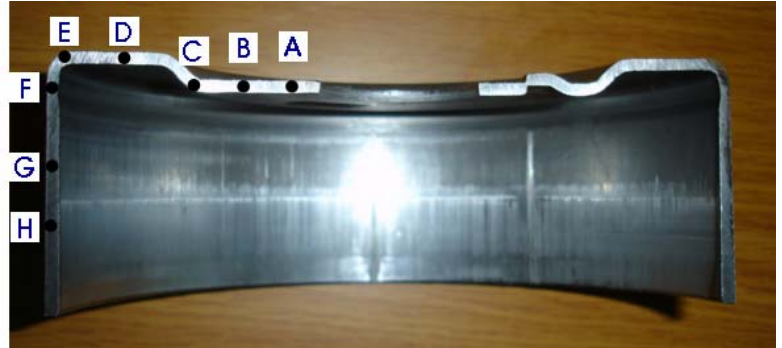

Fig. 11 Section view of hub perform and location of dimension measurement

Table 5: Comparison of thickness measurement between FE simulation and test product

\begin{tabular}{|c|c|c|c|}
\hline & $\begin{array}{c}\text { Result of FE } \\
\text { simulation }\end{array}$ & Result of Testing & DIFF \\
\hline A & 2.8530 & 2.95 & +0.0970 \\
\hline B & 2.8517 & 2.99 & +0.1383 \\
\hline C & 2.8546 & 2.80 & -0.0546 \\
\hline D & 2.8869 & 2.86 & -0.0269 \\
\hline E & 3.2 .125 & 3.10 & -0.1125 \\
\hline F & 2.7365 & 2.78 & 0.0435 \\
\hline G & 3.0213 & 3.00 & -0.0213 \\
\hline H & 3.1210 & 3.02 & -0.1010 \\
\hline
\end{tabular}

\section{ACKNOWLEDGEMENTS}

This work was supported by grant No.RT104-01-03 from the Regional Technology Innovation Program of the Ministry of Knowledge Economy(MKE)

\section{REFERENCES}

[1] Park, J. N., Kim, D. H. and Kim, B. M., "Experimental Investigation on the Flow Control of Hub Clutch for Automobile", Transaction of Materials Processing, Vol. 11, No. 5, pp. 430-438, 2002.

[2] Hussain, P. B., Cheon, J. S., Kwak, K. Y., Kim, S. Y. and Im, Y. T., "Simulation of Clutch-hub Forging Process using CAMPform,” Journal of Materials Processing Technology, Vol. 123, pp. 120-132, 2002.

[3] Yokoayma, Y. and Taguchi, G., "Business data Analysis: Experimental Regression Analysis,” Maruzen, 1975 